\title{
The Application of Artificial Intelligence Technology in Network Technology
}

\author{
Yanqing Liu \\ Network Information Center of Dalian Jiaotong University, Dalian City, Liaoning Province, 116028
}

Keywords: artificial intelligence technology; characteristic; network technology; application

\begin{abstract}
In this paper, the artificial intelligence application in network technology is associated with a theoretical practice. Based on the brief description of artificial intelligence, this paper analyzes the characteristics of artificial intelligence technology and existing problems in network technology. Moreover, it provides the specific application of artificial intelligence technology in network technology. Therefore, results show that the rational application of artificial intelligence technology in network technology can effectively ensure the safety of network technology, can also provide the basic guarantee for the continuous stability of network technology, and hopefully help the relevant units.
\end{abstract}

\section{Introduction}

Network technology has great significance to the development of a country's social and economic stability. It is also the main measure of the country's development level. Artificial intelligence technology has extremely strong fuzzy processing ability and learning ability. We apply it in the network technology will have great significance for the development of China's network infrastructure. Therefore, based on speculative practice, this paper analyzes the pertinent situation of artificial intelligence technology and its specific application for computer network technology.

\section{Overview of Artificial Intelligence Technology}

Artificial intelligence technology, is identified as AI technology internationally. It is a new kind of technical science. Artificial intelligence technology is very comprehensive and professional that integrate with computer technology, psychology, physiology, linguistics and so on. Advanced artificial intelligence technology can endow mechanical equipment with human activities and actions. And it can be utilized in the dangerous and complicated working environment to ensure people's safety. Artificial intelligence technology is a new research field. It is also the principal product under the development of network technology and computer chip technology. It can promote the system or equipment to simulate human's various characteristics, so that to have its human intelligence activities.

Artificial intelligence technology belongs to a multidisciplinary application technology. It can lead to closely link between two or more seemingly unrelated disciplines. The rapid progress of computer science and technology has laid a solid foundation for the application and development of artificial intelligence technology. The development of network technology also dependent on artificial intelligence technology.

\section{Research Importance of Artificial Intelligence Technology}

The development and application of artificial intelligence technology are the principal criterion to measure the advanced technology development in a country. Breakthroughs in diverse technologies, such as aviation and industrial development, are inseparable from artificial intelligence technology. It can realize the native state and the whole state of the system resources. At the same time, the information can be comprehensively analyzed and processed, which can provide users with the necessary resources and information anytime and anywhere. The artificial intelligence technology also has the excellent writing ability. It can integrate and analyze relevant 
information and resources. With this function, we can realize the resource sharing and transmission of different users. In particular, it can apply the distributed artificial intelligence thought of multi-agent writing and network management. This method will increase the network management efficiency. Therefore, it has obvious advantages to integrate artificial intelligence technology into network technology, which can effectively increase the learning ability of network technology. Moreover, it can promote the memory function of network technology and lay a solid foundation for the steady development of computer network technology.

\section{Characteristics of Artificial Intelligence Technology}

\subsection{Strong fuzzy processing ability.}

We integrate artificial intelligence technology into computer network technology can make fuzzy processing of many uncertain information and resources. Therefore, it can greatly improve the operability of network technology, improve the management level of uncertain information, and give full play to the advantages and values of network technology.

\subsection{Layering implementation.}

Apply artificial intelligence technology into computer network technology, we can realize personating the computer network system. In short, it can realize the enterprise management simulation. The superior leader can direct the subordinate leaders, and the subordinate leaders can manage the ordinary employees. The employee can supervise the behavior of the subordinate leaders, and the subordinate leaders can supervise the whole working process of the superior. Through coordination between superiors and subordinates, it is possible to provide a suitable environment for the safe and stable operation of computer network system [1].

\subsection{Strong learning ability.}

Learning ability is a part of the core characteristics of artificial intelligence technology. And it is also the core energy for the widely use of this technology. Integrate artificial intelligence technology into computer network system can greatly reduce the operation and application difficulty of computer network technology. For data and information processing hardness, we can perform the higher level management and prompt the best computer system operation.

\subsection{Few resource consumption, fast processing information speed.}

Apply the artificial intelligence technology into network technology can realize rapid analysis and internal processing of relevant data and information. It can effectively save users' access time and meet people's demands for speed and quality.

\section{Current Problems of Network Technology}

With the steady progress of science and technology in China, computer network technology is becoming more and more advanced. Its application scope is also expanding. People's network information security question put forward higher request. Network control technology and network monitoring technology are the main technologies to ensure data and information security in the computer network system. However, these two technologies have strong timeliness and comprehensiveness. The function application realization is directly related to the security of the entire network system. Therefore, based on the information acquisition and processing, through the irregular and discontinuous of computer network data, we can realize the logical analysis and processing of data and information. However, we cannot make the data and the information authenticity judgment. To extract really useful information from mass data, we need to achieve it through the intelligence of computer network technology. In recent years, the application of computer network technology has grown in depth and breadth. And it has grown higher requirements for network security management. The progress of software development technology and network crime increases constantly. If computer network technology does not have the ability 
of sensitive observation and quick reaction, it is difficult to assure the security of users and enterprises related information and resources.

A large number of applications at home and abroad indicate that in order to make full use of the data and information security in the computer network system, we must rely on artificial intelligence technology. Build a more secure network technology protection platform can quickly put forward effective and valuable information from the mass data repository. It is also the principal way to realize intelligent network application [2]. Then we can realize automatic data collection, timely diagnosis, performance and trend of fault diagnosis, etc. For example, when a computer network system fails, the system can respond quickly. And through the maintenance method during the database timely processing, we can greatly improve the system fault processing speed. Therefore, artificial intelligence technology can also establish a seamless network defense system and management system within the computer network system, effectively ensuring the security of relevant information and data in the system.

\section{The Application of Artificial Intelligence Technology in Network Technology}

As can be seen from the above analysis, artificial intelligence technology has obvious advantages in numerous aspects. However, there are a number of problems to be solved in the computer network system. Combining artificial intelligence technology and computer network system will have great significance to the continuous and stable development of network technology. The specific application is as follows:

\subsection{In network security management.}

Although computer network technology plays a very important role in the life quality improvement and the social productivity promotion, the problem of network security is also a major problem that currently affects many people. If we apply the artificial intelligence technology into computer network security management, we can effectively assure the security of data and information. It is mainly reflected in the following aspects:

Firstly, it can automatically process the junk mail. Through the artificial intelligence technology, the computer network system can accurately identify the junk mail in the system, and conduct automatic processing. For instance, in the normal operation of a computer network system, there is an email with sensitive information The artificial intelligence system can immediately carry on the whole process detection to this email, take corresponding security protection measures, and avoid this email into a computer network system. This can effectively ensure the security of user information. At present, this function of artificial intelligence technology in China has been fully applied in QQ mailbox and Sina mailbox, which has achieved excellent application results.

Secondly, it can automatically exclude the dangerous information. In the current development situation of the security management for the computer network system in China, the firewall is the top priority to protect the user's information. If the firewall technology is to be integrated into artificial intelligence technology, it can control and identify the applicable information. It can also blur the information that is uncertain. Once there is a risk, the firewall can automatically identify and eliminate it, guarantees the entire computer network security. For example, China's 360 Security Guard, Kingsoft antivirus and other established computer security protection systems all apply artificial intelligence technology [3].

Thirdly, it can detect network intrusion. Through the application of intrusion detection technology, we can analyze, classify and filter computer network data. Then we can deal with the analysis, classification and filtering data through the corresponding programming software. Then transfer the processing results to the computer directly, and provide users the actual situation of the network system operation. The problem is solved in time to ensure the security of data information. Network intrusion detection is a very complex and systematic process that needs various subjects' application. Artificial intelligence is one of the most critical technologies.

Through the above analysis, we can learn that the artificial intelligence technology integration in network technology can effectively make up some security holes in the operation of computer 
network technology. It can ensure the security of relevant data and information in a computer network system.

\subsection{In agent technology}

The structure diagram of Agent technology system is illustrated in figure 1.

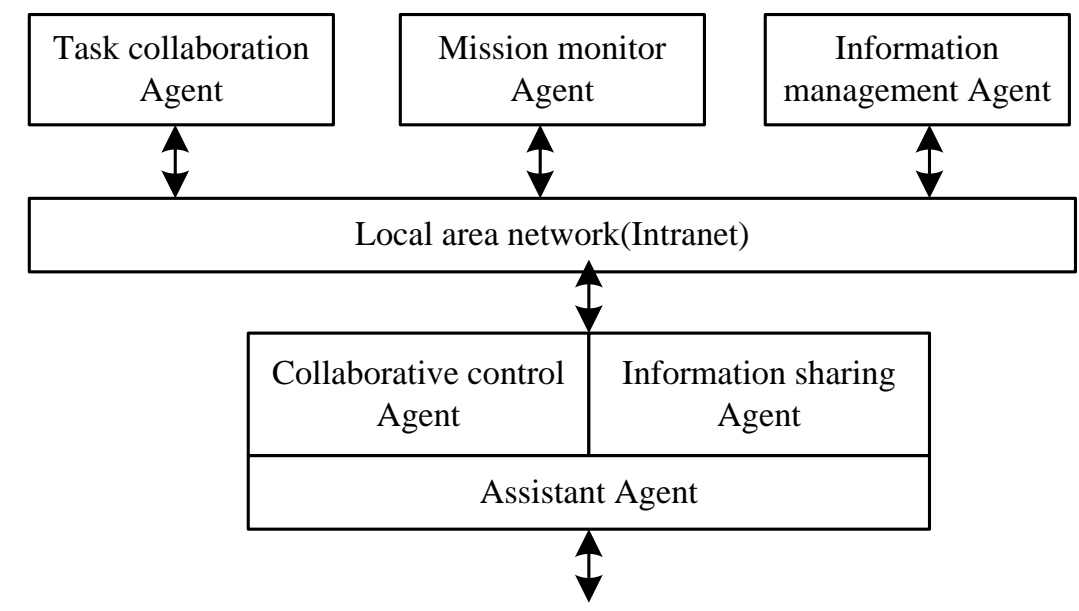

Figure 1. Structure diagrams of agent technology system

Agent technology of artificial intelligence is a type of advanced computer network technology. It can greatly improve the application level of network technology. In the agent application process of artificial intelligence, it requires the support of the knowledge domain library and interpreter. Information can be saved and processed through a complex and variable system. According to the self-defined criteria, agent technology of artificial intelligence can select the information that needed by the users and transmit the information to the designated location through the computer network system. This technology effectively meets the needs of users' personalization and diversification of network technology [4]. It can also integrate the useful information, form a new knowledge base, and then combine the corresponding fuzzy processing technology to provide the user with a comprehensive and whole-process navigation service.

\subsection{In system evaluation.}

Operational security evaluation of the computer network system is a very complex work. It needs the knowledge network library that built by telecom and artificial intelligence technology. Then we can conduct all-round and whole process supervision and evaluation. After that, it can provide real and effective data for the safety and stability evaluation of computer systems. Additional technologies have limitations, and artificial intelligence technology can effectively solve this problem [5].

Expert decision and support laid a solid foundation for the artificial intelligence technology application in network technology. For example: We integrate the decision-making and knowledge of experts in one field into a database. When the problem is discovered, the opinions and countermeasures can be processed through the database output. It will greatly improve the effectiveness of network technology application.

\section{Summary}

In conclusion, based on the theoretical practice, this paper studies the application of artificial intelligence technology in network technology. The research results show that artificial intelligence technology has immense significance for the development of a country's social economy. It is also a major method to form global competitiveness. A large number of application examples show that we integrate artificial intelligence technology into network technology can realize the development requirement of China's network industry in the new era, and it is worthy to extensive the 
application.

\section{References}

[1] Sheng Xu. Artificial Intelligence and Its Application in Computer Network Technology [J]. Telecom World, 2016(6):87-87.

[2] Wang Yanxian. The Application of Artificial Intelligence in the Networks [J]. Information Technology and Informatization, 2015(8):84-85.

[3] Wang Zhihong, Yang Zhen. Research on Artificial Intelligence Technology and the Future Intelligent Information Service Architecture [J]. Telecommunications Science, 2017, 33(5):1-11.

[4] Gu Shoujun, Wang Haiyong. The Artificial Intelligence Application in Computer Network Technology of Big Data Era [J]. Practical Electronics, 2017(6):30-30.

[5] Xu Ying. Brief Discussion on the Application of Artificial Intelligence in Computer Network Technology [J]. Digital Space, 2017(5):8-8.

[6] Lv Zhiheng. Application Analysis of Artificial Intelligence in Computer Network System Management and Evaluation [J]. Digital Space, 2017(5):103-103. 